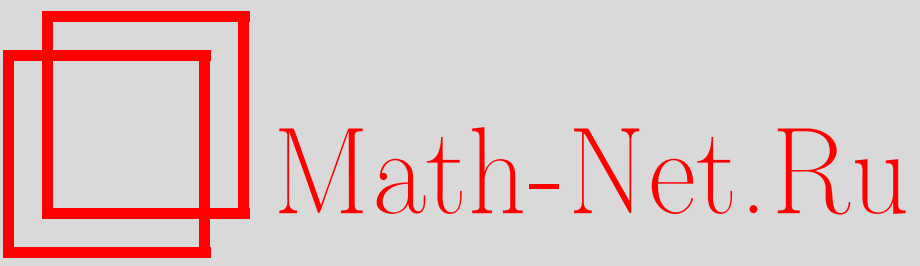

Б. В. Аверин, О тепловой устойчивости многослойных плоских стенок при нагреве внутренними источниками, зависящими от температуры, Becmн. Cам. гос. техн. ун-та. Сер. Физ.-мат. науки, 2009, выпуск 2(), 177-185

DOI: https://doi.org/10.14498/vsgtu701

Использование Общероссийского математического портала Math-Net.Ru подразумевает, что вы прочитали и согласны с пользовательским соглашением

http: //www. mathnet.ru/rus/agreement

Параметры загрузки:

IP : 52.205 .19 .152

26 апреля 2023 г., 15:34:15 
УДК 517.958:[536.2+539.219.3]

\section{О ТЕПЛОВОЙ УСТОЙЧИВОСТИ МНОГОСЛОЙНЫХ ПЛОСКИХ СТЕНОК ПРИ НАГРЕВЕ ВНУТРЕННИМИ ИСТОЧНИКАМИ, ЗАВИСЯЩИМИ ОТ ТЕМПЕРАТУРЫ}

\section{Б. В. Аверин}

Сызранский филиал Самарского государственного технического университета, 446001, Самарская обл., Сызрань, ул. Советская, 45.

E-mail: totig@yandex.ru

Приводится последовательность получения аналитического решения стачионарной нелинейной задачи теплопроводности для многослойной плоской стенки с зависящими от температуры внутренними источниками теплоты. На основе анализа полученного решения применительно $к$ двухслойной стенке получены кривые, позволяющие при конкретных исходных данных определить мощность источников теплоты, при которых происходит неограниченное возрастание температуры (тепловой взрыв).

Ключевые слова: аналитические решения, нелинейные задачи, многослойные конструкции, внутренние источники теплоты, тепловой взрыв.

Задачи, связанные с нелинейным нагревом многослойных конструкций от действия внутренних источников теплоты, имеют важный научный и практический интерес [1-6]. При решении этих задач важнейшей проблемой является определение предельной мощности источников, при которых количество получаемой от них теплоты не может быть полностью отведено от конструкции при заданных граничных условиях теплообмена. Такие режимы нагрева приводят к неограниченному возрастанию температуры в конструкции и в конечном итоге - к её тепловому разрушению. Для определения мощности таких источников теплоты необходимо иметь аналитическое решение соответствующей краевой задачи.

Эффективным методом решения задач теплопроводности для многослойных конструкций является метод, основанный на применении обобщенных функций. При этом многослойная система заменяется однослойной, но с переменными (разрывными) физическими свойствами среды, которые описываются с помощью единичных характеристических (асимметричных) функций. Простота такого метода в том, что в данном случае нет необходимости специального выполнения условий сопряжения. Благодаря особым свойствам асимметричной функции, условиям идеального термомеханического контакта между слоями удается удовлетворить непосредственно в уравнении.

Рассмотрим многослойную плоскую стенку, у которой на поверхности $x=x_{0}=0$ задана температура окружающей среды $t_{1}$, а на поверхности $x=x_{n}$ - температура $t_{2}$. Предположим, что в пределах каждого слоя удельные мощности тепловых источников аппроксимируются линейными функциями температуры

$$
q_{v_{i}}=a_{i}+b_{i} T
$$

где $a_{i}=\omega_{0 i}, b_{i}=\omega_{0 i} \beta_{i} ; \omega_{0 i}-$ удельная мощность постоянно действующего

Борис Викторович Аверин (к.т.н.), доцент, каф. общетеоретических дисциплин. 


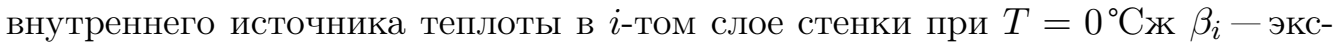
периментальная постоянная для $i$-того слоя стенки.

Представим коэффициент теплопроводности и удельную мощность внутреннего источника теплоты для многослойной стенки как единые целые в виде

$$
\begin{gathered}
\lambda(x)=\lambda_{1}+\sum_{i=1}^{n-1}\left(\lambda_{i+1}-\lambda_{i}\right) H\left(x-x_{i}\right) ; \\
q_{v}(x, T)=a(x)+b(x) T
\end{gathered}
$$

где

$$
\begin{aligned}
& a(x)=a_{1}+\sum_{i=1}^{n-1}\left(a_{i+1}-a_{i}\right) H\left(x-x_{i}\right), \\
& b(x)=b_{1}+\sum_{i=1}^{n-1}\left(b_{i+1}-b_{i}\right) H\left(x-x_{i}\right),
\end{aligned}
$$

$H\left(x-x_{i}\right)=\left\{\begin{array}{ll}0, & \text { если } x<x_{i}, \\ 1, & \text { если } x \geqslant x_{i}\end{array}\right.$ - асимметричная единичная функция.

Дифференциальное уравнение теплопроводности для определения стационарного температурного поля в многослойной конструкции с нелинейными внутренними источниками теплоты с учётом (1)-(3) в этом случае будет иметь вид

$$
\frac{d}{d x}\left[\lambda(x) \frac{d T(x)}{d x}\right]+b(x) T+a(x)=0 .
$$

Перейдём в (4) от переменной $x$ к новой независимой переменной $z$ по формуле

$$
z=\int_{0}^{x} \sqrt{\frac{b(\xi)}{\lambda(\xi)}} d \xi=\sqrt{\frac{b_{1}}{\lambda_{1}}} x+\sum_{i=1}^{n-1}\left(\sqrt{\frac{b_{i+1}}{\lambda_{i+1}}}-\sqrt{\frac{b_{i}}{\lambda_{i}}}\right) H\left(x-x_{i}\right) .
$$

Тогда уравнение (4) в новой переменной (5) преобразуется к неоднородному дифференциальному уравнению с постоянными и сингулярными коэффициентами вида

$$
\frac{d^{2} T}{d z^{2}}+T(z)=-\left.\sum_{i=1}^{n-1}\left(\sqrt{\frac{\lambda_{i+1} b_{i+1}}{\lambda_{i} b_{i}}}-1\right) \frac{d T}{d z}\right|_{z=z_{i}} \delta\left(z-z_{i}\right)-\frac{a(z)}{b(z)},
$$

где $\delta\left(z-z_{i}\right)$ - дельта-функция Дирака.

Общее решение уравнения (6) с помощью метода вариации произвольных постоянных можно представить в виде

$$
T(z)=C_{1} \sin z+C_{2} \cos z+D_{1}(z) \sin z-D_{2}(z) \cos z,
$$

где

$$
D_{1}(z)=\int_{0}^{z} f(z) \cos z d z, \quad D_{2}(z)=\int_{0}^{z} f(z) \sin z d z
$$




$$
f(z)=-\left.\sum_{i=1}^{n-1}\left(\sqrt{\frac{\lambda_{i+1} b_{i+1}}{\lambda_{i} b_{i}}}-1\right) \frac{d T}{d z}\right|_{z=z_{i}}-\frac{a(z)}{b(z)} .
$$

После вычисления соответствующих интегралов общее решение (7) запишется так:

$$
\begin{aligned}
T(z)=C_{1} \sin z+C_{2} & \cos z-\frac{a_{1}}{b_{1}}-\sum_{i=1}^{n-1}\left\{\left(\frac{a_{i+1}}{b_{i+1}}-\frac{a_{i}}{b_{1}}\right)\left[1-\cos \left(z-z_{i}\right)\right]+\right. \\
+ & \left.\left.\left(\sqrt{\frac{\lambda_{i+1} b_{i+1}}{\lambda_{i} b_{i}}}-1\right) \frac{d T}{d z}\right|_{z=z_{i}} \sin \left(z-z_{i}\right)\right\} H\left(z-z_{i}\right) .
\end{aligned}
$$

Неизвестные производные $\left.\frac{d T}{d z}\right|_{z=z_{i}}$ в (8) представим в виде

$$
\left.\frac{d T}{d z}\right|_{z=z_{i}}=D_{1}^{i} C_{1}-D_{2}^{i} C_{2}-D_{3}^{i}
$$

где $D_{1}^{i}, D_{2}^{i}, D_{3}^{i}$ определяются из следующих рекуррентных соотношений:

$$
\begin{gathered}
D_{1}^{j}=\sqrt{\frac{\lambda_{j} b_{j}}{\lambda_{j+1} b_{j+1}}}\left[\cos z_{j}-\sum_{i=1}^{j-1}\left(\sqrt{\frac{\lambda_{i+1} b_{i+1}}{\lambda_{i} b_{i}}}-1\right) D_{1}^{i} \cos \left(z_{j}-z_{i}\right)\right] \\
D_{2}^{j}=\sqrt{\frac{\lambda_{j} b_{j}}{\lambda_{j+1} b_{j+1}}}\left[\sin z_{j}-\sum_{i=1}^{j-1}\left(\sqrt{\frac{\lambda_{i+1} b_{i+1}}{\lambda_{i} b_{i}}}-1\right) D_{2}^{i} \cos \left(z_{j}-z_{i}\right)\right] \\
D_{3}^{j}=\sqrt{\frac{\lambda_{j} b_{j}}{\lambda_{j+1} b_{j+1}}}\left[\sum_{i=1}^{j-1}\left(\sqrt{\frac{a_{i+1}}{b_{i+1}}}-\frac{a_{i}}{b_{i}}\right) \sin \left(z_{j}-z_{i}\right)-\right. \\
\left.-\sum_{i=1}^{j-1}\left(\sqrt{\frac{\lambda_{i+1} b_{i+1}}{\lambda_{i} b_{i}}}-1\right) D_{3}^{i} \cos \left(z_{j}-z_{i}\right)\right],
\end{gathered}
$$

где $j \equiv 1,2, \ldots, n-1$.

После подстановки (9) в (8), получим

$$
\begin{gathered}
T(z)=\left[\sin z-\sum_{i=1}^{n-1}\left(\sqrt{\frac{\lambda_{i+1} b_{i+1}}{\lambda_{i} b_{i}}}-1\right) D_{1}^{i} \sin \left(z-z_{i}\right) H\left(z-z_{i}\right)\right] C_{1}+ \\
+\left[\cos z+\sum_{i=1}^{n-1}\left(\sqrt{\frac{\lambda_{i+1} b_{i+1}}{\lambda_{i} b_{i}}}-1\right) D_{2}^{i} \sin \left(z-z_{i}\right) H\left(z-z_{i}\right)\right] C_{2}- \\
-\frac{a_{1}}{b_{1}}-\sum_{i=1}^{n-1}\left(\sqrt{\frac{a_{i+1}}{b_{i+1}}}-\frac{a_{i}}{b_{i}}\right)\left[1-\cos \left(z-z_{i}\right)\right] H\left(z-z_{i}\right)+ \\
+\sum_{i=1}^{n-1}\left(\sqrt{\frac{\lambda_{i+1} b_{i+1}}{\lambda_{i} b_{i}}}-1\right) D_{3}^{i} \sin \left(z-z_{i}\right) H\left(z-z_{i}\right) .
\end{gathered}
$$


Постоянные интегрирования $C_{1}$ и $C_{2}$ находим из граничных условий

$$
T=t_{1}, \quad \text { при } z=0 ; \quad T=t_{2}, \quad \text { при } z=z_{n},
$$

где

$$
z_{n}=\sqrt{\frac{b_{1}}{\lambda_{1}}} h+\sum_{i=1}^{n-1}\left(\sqrt{\frac{b_{i+1}}{\lambda_{i+1}}}-\sqrt{\frac{b_{i}}{\lambda_{i}}}\right)\left(h-x_{i}\right) ;
$$

$h$ - толщина $n$-слойной стенки.

Подставляя (13) в (14), получаем

$$
C_{1}=\frac{A}{R}, \quad C_{2}=t_{1}+\frac{a_{1}}{b_{1}} ;
$$

где

$$
\begin{aligned}
A=t_{2}+\frac{a_{1}}{b_{1}} & +\sum_{i=1}^{n-1}\left(\frac{a_{i+1}}{b_{i+1}}-\frac{a_{i}}{b_{i}}\right)\left[1-\cos \left(z_{n}-z_{i}\right)\right]- \\
& -\sum_{i=1}^{n-1}\left(\sqrt{\frac{\lambda_{i+1} b_{i+1}}{\lambda_{i} b_{i}}}-1\right) D_{3}^{i} \sin \left(z_{n}-z_{i}\right) H\left(z-z_{i}\right)- \\
- & {\left[\cos z_{n}+\sum_{i=1}^{n-1}\left(\sqrt{\frac{\lambda_{i+1} b_{i+1}}{\lambda_{i} b_{i}}}-1\right) D_{2}^{i} \sin \left(z_{n}-z_{i}\right)\right]\left(t_{1}+\frac{a_{1}}{b_{1}}\right) ; } \\
R & =\sin z_{n}-\sum_{i=1}^{n-1}\left(\sqrt{\frac{\lambda_{i+1} b_{i+1}}{\lambda_{i} b_{i}}}-1\right) D_{1}^{i} \sin \left(z_{n}-z_{i}\right) .
\end{aligned}
$$

После подстановки (15) в (13) с учётом (16) и (17) получим

$$
T(z)=\frac{A \varphi_{1}(z)}{R}+\varphi_{2}(z),
$$

где

$$
\begin{gathered}
\varphi_{1}(z)=\sin z-\sum_{i=1}^{n-1}\left(\sqrt{\frac{\lambda_{i+1} b_{i+1}}{\lambda_{i} b_{i}}}-1\right) D_{1}^{i} \sin \left(z-z_{i}\right) H\left(z-z_{i}\right) \\
\varphi_{2}(z)=\left(t_{C_{1}}+\frac{a_{1}}{b_{1}}\right)\left[\cos z+\sum_{i=1}^{n-1}\left(\sqrt{\frac{\lambda_{i+1} b_{i+1}}{\lambda_{i} b_{i}}}-1\right)\right] D_{2}^{i} \sin \left(z-z_{i}\right) H\left(z-z_{i}\right)- \\
-\frac{a_{1}}{b_{1}}-\sum_{i=1}^{n-1}\left(\frac{a_{i+1}}{b_{i+1}}-\frac{a_{i}}{b_{i}}\right)\left[\left(1-\cos \left(z-z_{i}\right)\right] H\left(z-z_{i}\right)+\right. \\
+\sum_{i=1}^{n-1}\left(\sqrt{\frac{\lambda_{i+1} b_{i+1}}{\lambda_{i} b_{i}}}-1\right) D_{3}^{i} \sin \left(z-z_{i}\right) H\left(z-z_{i}\right) .
\end{gathered}
$$


Рассмотрим знаменатель $R$ в формуле (18). Например, для двухслойной стенки, с учётом обозначений, принятых в (1), его можно представить следующим образом:

$$
R=\sin z_{2}-\left(\sqrt{\frac{\lambda_{i} b_{i}}{\lambda_{2} b_{2}}}-1\right) \cos z_{1} \sin \left(z_{2}-z_{1}\right)
$$

или в таком виде:

$$
R=\sin z_{2}-\left(\sqrt{\frac{\lambda_{i} \omega_{0_{1}} \beta_{1}}{\lambda_{2} \omega_{0_{2}} \beta_{2}}}-1\right) \cos z_{1} \sin \left(z_{2}-z_{1}\right),
$$

где $z_{1}=B_{1} h_{1}, z_{2}=B_{1} h_{1}+B_{2} h_{2}, B_{1}=\sqrt{\frac{\omega_{0_{1}} \beta_{1}}{\lambda_{1}}}, B_{2}=\sqrt{\frac{\omega_{0_{2}} \beta_{2}}{\lambda_{2}}} ; h_{1}, h_{2}$ - толщина первого и второго слоев.

Запишем (20) следующим образом:

$$
R=\sin \left[\left(z_{2}-z_{1}\right) N_{2}\right]-\left(1-K_{\varepsilon_{1}}\right) \cos \left[\left(z_{2}-z_{1}\right) N_{1}\right] \sin \left[\left(z_{2}-z_{1}\right) N_{3}\right],
$$

где

$$
\begin{gathered}
N_{1}=\frac{z_{1}}{z_{2}-z_{1}}, \quad N_{2}=\frac{z_{2}}{z_{2}-z_{1}}, \quad N_{3}=\frac{z_{2}-z_{1}}{z_{2}-z_{1}} ; \\
K_{\varepsilon_{1}}=\sqrt{\frac{\lambda_{1} \omega_{0_{1}} \beta_{1}}{\lambda_{2} \omega_{0_{2}} \beta_{2}}}, \quad z_{2}-z_{1}=B_{2} h_{2} .
\end{gathered}
$$

Обозначим

$$
B_{2} h_{2}=\mu_{1} .
$$

Соотношение (21) с учётом (22) примет вид

$$
R=\sin \mu N_{2}-\left(1-K_{\varepsilon_{1}}\right) \cos \mu N_{1} \sin \mu .
$$

Рассмотрим условия, при которых знаменатель формулы (18) обращается в ноль и, следовательно, температура принимает бесконечное значение:

$$
\sin \mu N_{2}-\left(1-K_{\varepsilon_{1}}\right) \cos \mu N_{1} \sin \mu=0 .
$$

Выполнив преобразования в (24) с учётом (22), получим

$$
\sin \left[\mu_{1}\left(1+K_{\varepsilon_{1}} \frac{\lambda_{2} h_{1}}{\lambda_{1} h_{2}}\right) N_{2}\right]-\left(1-K_{\varepsilon_{1}}\right) \cos \left[\mu_{1}\left(K_{\varepsilon_{1}} \frac{\lambda_{2} h_{1}}{\lambda_{1} h_{2}}\right) N_{2}\right] \sin \mu_{1}=0 .
$$

Таким образом, задача о критических тепловых режимах, приводящих к тепловому взрыву, в двухслойной стенке свелась к решению трансцендентного уравнения $(25)$, т. е. к отысканию первого корня $\mu_{1}$ при различных значениях безразмерных параметров $K_{\varepsilon_{1}}$ и $\eta=\frac{\lambda_{2} h_{1}}{\lambda_{1} h_{2}}$. Из $(22)$ найдём, что $\omega_{0_{2, \max }}=\frac{\mu_{1}^{2} \lambda_{2}}{\beta_{2} h_{2}^{2}}$.

Если удельная мощность постоянно действующего внутреннего источника теплоты во втором слое при известном внутреннем источнике теплоты в 
первом слое превысит $\omega_{0_{2, \max }}$, то тепловой поток с поверхностей двухслойной стенки при заданных $t_{1}$ и $t_{2}$ нельзя отвести, и произойдёт разрушение материалов конструкции.

На рис. 1 представлены графики зависимости $\mu_{1}=f\left(\eta, K_{\varepsilon_{1}}\right)$, отделяющие область, в которой стационарное распределение температуры возможно, от области неограниченного возрастания температуры. Проиллюстрируем их применение при следующих исходных данных: $\lambda_{1}=0,065 \mathrm{BT} / \mathrm{м} \cdot \mathrm{K}, \lambda_{2}=$ $=0,32 \mathrm{BT} / \mathrm{m} \cdot \mathrm{K}, \omega_{0_{1}}=11,68 \mathrm{\kappa BT} / \mathrm{m}^{3}, \omega_{0_{2}}=161,7 \mathrm{\kappa BT} / \mathrm{m}^{3}, \beta_{1}=0,01 \mathrm{~K}^{-1}$, $\beta_{2}=0,1 \mathrm{~K}^{-1}, h_{1}=30 \cdot 10^{-3} \mathrm{M}, h_{2}=7,5 \cdot 10^{-3} \mathrm{M}$.

Определим параметры $\eta$ и $K_{\varepsilon_{1}}$ :

$$
\eta=\frac{\lambda_{2}}{\lambda_{1}} \frac{h_{1}}{h_{2}}=\frac{0,32}{0,065} \frac{30 \cdot 10^{-3}}{7,5 \cdot 10^{-3}} \approx 19,6923 .
$$

Так как $\eta$ изменяется в пределах $0 \leqslant \eta \leqslant \pi$, то представим 19,6923 как $6 \pi+$ $+0,268 \pi$. Отбрасывая количество периодов $6 \pi$, окончательно получаем, что $\eta \approx 0,268 \pi \approx 0,843$ и

$$
K_{\varepsilon_{1}}=\sqrt{\frac{\omega_{0_{1}} \beta_{1} \lambda_{1}}{\omega_{0_{2}} \beta_{2} \lambda_{2}}}=\sqrt{\frac{0,01 \cdot 0,065}{0,1 \cdot 0,32}} \sqrt{\frac{11,68}{161,7}} \approx 0,4 .
$$

Используя найденные $\eta$ и $K_{\varepsilon_{1}}$, из графика находим $\mu_{1} \approx 2,1$. Тогда критическая мощность внутреннего источника теплоты, действующего во втором слое, определяется выражением

$$
\omega_{0, \max }=\frac{\mu_{1}^{2} \lambda_{2}}{\beta_{2} h_{2}^{2}} \approx \frac{4,41 \cdot 0,32 \cdot 10^{6}}{0,1 \cdot 56,25} \approx 250 \mathrm{\kappa BT} / \mathrm{m}^{3} .
$$

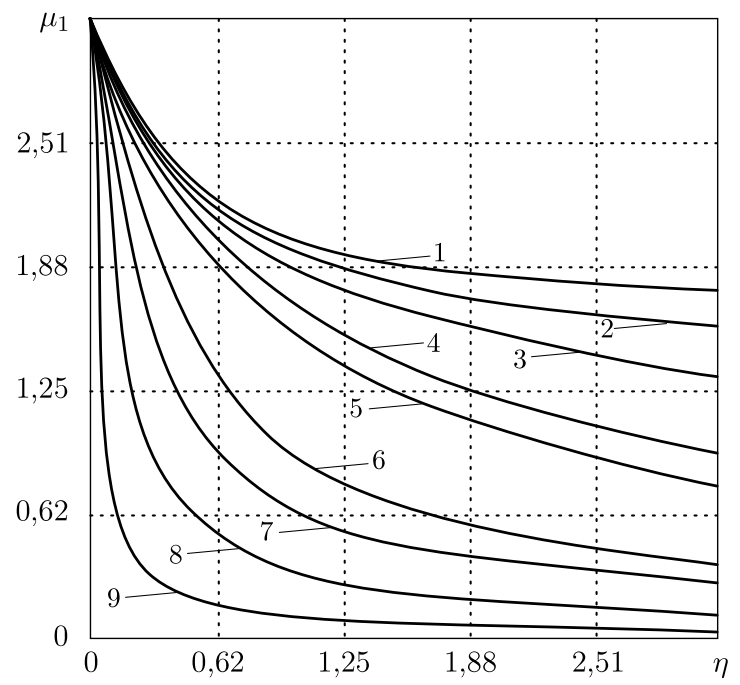

Рис. 1. Критические кривые, при которых знаменатель $R$ в формуле (18) принимает нулевые значения (выполняются условия теплового взрыва); цифры: $1-K_{\varepsilon}=0,001,2-K_{\varepsilon}=0,3,3-K_{\varepsilon}=0,5,4-K_{\varepsilon}=0,8,5-K_{\varepsilon}=1$, $6-K_{\varepsilon}=2,7-K_{\varepsilon}=3,8-K_{\varepsilon}=6,9-K_{\varepsilon}=10$ 
Критическую мощность внутреннего источника теплоты, действующего в первом слое, найдём из соотношения

$$
\omega_{0_{1, \max }}=0,072 \omega_{0_{2, \max }} \approx 18 \mathrm{KBT} / \mathrm{M}^{3} .
$$

Приравняем к нулю выражение (19):

$$
\sin z_{2}+\left(\sqrt{\frac{\lambda_{1} b_{1}}{\lambda_{2} b_{2}}}-1\right) \cos z_{1} \sin \left(z_{2}-z_{1}\right)=0
$$

Преобразуем его к виду

$$
\operatorname{tg} z_{2}=\frac{\sqrt{\frac{\lambda_{1} b_{1}}{\lambda_{2} b_{2}}}-1}{\sqrt{\frac{\lambda_{1} b_{1}}{\lambda_{2} b_{2}}}+\operatorname{tg}^{2} z_{1}} \operatorname{tg} z_{1}
$$

откуда

$$
z_{2}=\operatorname{arctg}\left[\frac{\sqrt{\frac{\lambda_{1} b_{1}}{\lambda_{2} b_{2}}}}{\sqrt{\frac{\lambda_{1} b_{1}}{\lambda_{2} b_{2}}}+\operatorname{tg}^{2} z_{1}} \operatorname{tg} z_{1}\right]+\pi \text {. }
$$

Учитывая, что

$$
z_{1}=h_{1} \sqrt{\frac{\omega_{0_{1}} \beta_{1}}{\lambda_{1}}}, \quad z_{2}=h_{1} \sqrt{\frac{\omega_{0_{1}} \beta_{1}}{\lambda_{1}}}+h_{2} \sqrt{\frac{\omega_{0_{2}} \beta_{2}}{\lambda_{2}}},
$$

выражению (27) можно придать такой вид:

$$
\frac{\operatorname{tg} h_{1} \sqrt{\frac{\omega_{0_{1}} \beta_{1}}{\lambda_{1}}}}{\operatorname{tg} h_{2} \sqrt{\frac{\omega_{0_{2}} \beta_{2}}{\lambda_{2}}}}=-\sqrt{\frac{\lambda_{1} \omega_{0_{1}} \beta_{2}}{\lambda_{2} \omega_{0_{2}} \beta_{2}}} .
$$

Откуда получаем расчётную формулу для определения критической толщины первого слоя стенки, если известна толщина второго слоя:

$$
h_{1 \text { кр }}=\sqrt{\frac{\lambda_{1}}{\omega_{0} \beta_{1}}}\left\{\operatorname{arctg}\left[-K_{\varepsilon_{1}} \operatorname{tg} h_{2} \sqrt{\frac{\omega_{0_{2}} \beta_{2}}{\lambda_{2}}}\right]+\pi\right\},
$$

и наоборот

$$
h_{2 \text { кр }}=\sqrt{\frac{\lambda_{2}}{\omega_{0} \beta_{1}}}\left\{\operatorname{arctg}\left[-K_{\varepsilon_{1}} \operatorname{tg} h_{1} \sqrt{\frac{\omega_{0_{1}} \beta_{1}}{\lambda_{1}}}\right]+\pi\right\} .
$$

Для трёхслойной стенки формула (17) будет иметь вид

$$
\begin{aligned}
R=\sin z_{3}+ & \left(\sqrt{\frac{\omega_{0_{1}} \lambda_{1} \beta_{1}}{\omega_{0_{2}} \lambda_{2} \beta_{2}}}-1\right) \cos z_{1} \sin \left(z_{3}-z_{1}\right)+ \\
+\left(\sqrt{\frac{\omega_{0_{2}} \lambda_{2} \beta_{2}}{\omega_{0_{3}} \lambda_{3} \beta_{3}}}-1\right) & {\left[\cos z_{2}+\left(\sqrt{\frac{\omega_{0_{1}} \lambda_{1} \beta_{1}}{\omega_{0_{2}} \lambda_{2} \beta_{2}}}-1\right) \times\right.} \\
& \left.\times \cos z_{1} \cos \left(z_{2}-z_{1}\right)\right] \sin \left(z_{3}-z_{2}\right)=0,
\end{aligned}
$$


где $z_{3}=B_{1} h_{1}+B_{2} h_{2}+B_{3} h_{3}, h_{3}$ - толщина третьего слоя, $B_{3}=\sqrt{\frac{\omega_{0_{3}} \beta_{3}}{\lambda_{3}}}$.

Рассмотрим частный случай, когда трёхслойная стенка представляет собой симметричную конструкцию. Разделив и умножив аргументы у тригонометрических функций в $(32)$ на $\left(z_{2}-z_{1}\right)=B_{2} h_{2}$, после несложных преобразований приходим к следующему характеристическому уравнению:

$$
\begin{aligned}
\sin \left[\mu _ { 1 } \left(1+K_{\varepsilon_{1}} \eta+\right.\right. & \left.\left.\frac{1}{K_{\varepsilon_{2}}} \eta\right)\right]-\left(1-K_{\varepsilon_{1}}\right) \cos \left(\mu_{1} K_{\varepsilon_{1}} \eta\right) \sin \left[\mu_{1}\left(1+\frac{1}{K_{\varepsilon_{2}}} \eta\right)\right]- \\
& -\left(1-K_{\varepsilon_{2}}\right)\left\{\cos \left[\mu_{1}\left(1+K_{\varepsilon_{1}} \eta\right)\right]-\right. \\
& \left.\left.-\left(1-K_{\varepsilon_{1}}\right) \cos \left(\mu_{1} K_{\varepsilon_{1}} \eta\right) \cos \mu_{1}\right)\right\} \sin \left(\mu_{1} \frac{1}{K_{\varepsilon_{2}}} \eta\right)=0
\end{aligned}
$$

где $K_{\varepsilon_{2}}=\sqrt{\frac{\omega_{0_{2}} \lambda_{2} \beta_{2}}{\omega_{0_{3}} \lambda_{3} \beta_{3}}}$.

Таким образом, задача о критических тепловых режимах в трёхслойной стенке свелась к отысканию методом последовательных приближений корня $\mu_{1}$ в зависимости от безразмерных параметров $K_{\varepsilon_{1}}, K_{\varepsilon_{2}}$ и $\eta$.

На рис. 2 предоставлены графики зависимости $\mu_{1}=f\left(\eta, K_{\varepsilon_{1}}, K_{\varepsilon_{2}}\right)$. Как и для двухслойной стенки, если удельная мощность постоянного действующего внутреннего источника теплоты во втором слое превысит $\omega_{\varepsilon_{2}, \max }$, то произойдет разрушение материалов стенки.

Таким образом, графики, представленные на рис. 1 и 2 , позволяют уже на стадии проектной разработки прогнозировать критические тепловые режимы и задавать такие значения внутренних источников и геометрические размеры конструкций, которые обеспечивают их тепловую устойчивость.

Аналогично решается задача и для $n$-слойных плоских стенок.

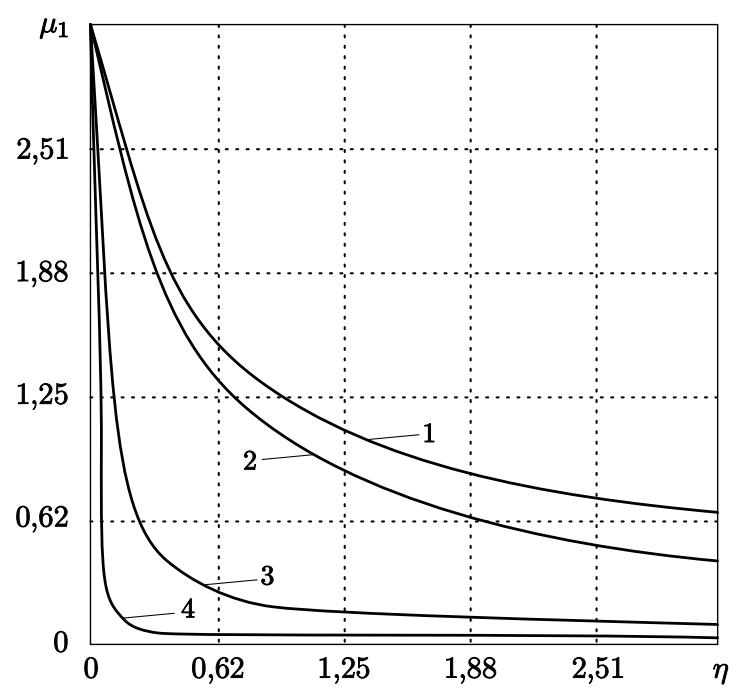

Рис. 2. Критические кривые, отделяющие довзрывную область от области теплового взрыва для трёхслойного РПУ. Кривые 1-4 соответствуют следующим значениям параметров $K_{\varepsilon_{1}}$ и $K_{\varepsilon_{2}}: 1-0,001 \leqslant K_{\varepsilon_{1}} \leqslant 0,1$, $10 \leqslant K_{\varepsilon_{2}} \leqslant 1000 ; 2-0,01 \leqslant K_{\varepsilon_{1}} \leqslant 1, K_{\varepsilon_{2}}=1 ; 3-0,01 \leqslant K_{\varepsilon_{1}} \leqslant 10, K_{\varepsilon_{2}}=0,1 ;$ $4-1 \leqslant K_{\varepsilon_{1}} \leqslant 100, K_{\varepsilon_{2}}=0,01$ 


\section{БИБЛИОГРАФИЧЕСКИЙ СПИСОК}

1. Аверин Б. В. Математическое моделирование внутренних источников теплоты, температурных полей и термических напряжений в многослойных радиопрозрачных укрытиях мощных передающих антенн: Автореф. дисс. ... канд. техн. наук, Моск. госуд. акад. тонкой хим. технол. / М., 1999. - 20 с.

2. Аверин Б.В., Колотилкин Д.И., Мелехина Н. М. К вопросу о законе распределения тепловых источников в многослойных РПУ при зависимости электрофизических свойств от температуры // Вопросъ специалъной радиоэлектроники. Сер. ТTA, 1990. - № 2(47). C. $46-61$.

3. Аверин Б. В., Колотилкин Д.И. Исследование тепловой устойчивости многослойных диэлектриков при их нагреве мощным электромагнитным СВЧ-полем / В сб.: Aвтоматизация технологических процессов и производств. Точность, качество и надёжность конструкиий и технических систем: Тез. докл. межвуз. научно-практ. семин.выставки. - Сызрань, 1997. - С. 47-48.

4. Гулабяни Л. А. Теплофизические основы проектирования ограждающих конструкций радиотехнических комплексов с высоким уровнем излучаемой мощности: Автореф. дисс. ... докт. тех. наук, НИИСФ / М., 1984. - 45 с.

5. Брыков С.И., Килъкеев Р.Ш., Ругинеи, Р. Г. Эффект нелинейного разогрева диэлектрика в СВЧ электромагнитном поле. - Минск: Ред. ИФЖ, 1987. - Деп. В ВИНИТИ 06.04.87. - Рег. № 2727-В.

6. Стефанюк E. B., Кудинов B. A. Аналитические решения задач теплопроводности при переменных во времени коэффициентах теплоотдачи // Вестн. Сам. гос. техн. ун-та. Сер. Физ.-мат. науки, 2008. - № 2(17). - С. 171-184.

Поступила в редакцию 08/VII/2009; в окончательном варианте - 21/X/2009.

MSC: 80A20, 35A09

\section{ON THERMAL STABILITY OF MULTI LAYER PLANE WALLS WHEN HEATING BY TEMPERATURE-DEPENDENT INTERNAL HEAT SOURCES}

\section{B. V. Averin}

Syzran' Branch of Samara State Technical University, 45, Sovetskaya str., Syzran', Samara region, 446001.

E-mail: totig@yandex.ru

Sequence of obtaining of analytical solution for a stationary non-linear problem of thermal conductivity for a multi layer plane wall with temperature-dependent internal heat source is presented. Curves are plotted that allow to separated the region of stationary temperature distribution from region of unrestricted growth of temperature (thermal explosion region) are plotting.

Key words: analytical solution, non-linear problem, multi layer design, internal heat source, thermal explosion.

Original article submitted 08/VII/2009; revision submitted $21 / \mathrm{X} / 2009$.

Boris V. Averin (Ph. D. (Techn.)), Associate Professor, Dept. of General Theoretical Discipline. 